\title{
Correction: FLASH-Radiotherapy: A Potential Innovation Driver in Radiation Therapy
}

\author{
[J. Korean Phys. Soc. 77, 357 (2020)] \\ DOI: $10.3938 / j k p s .77 .357$ \\ Young-Seok SEO \\ Department of Radiation Oncology, Chungbuk National University Hospital, Cheongju 28644, Korea \\ Woo-Yoon PARK* \\ Department of Radiation Oncology, Chungbuk National University Hospital, Cheongju 28644, Korea and \\ Department of Radiation Oncology, Chungbuk National University College of Medicine, Cheongju 28644, Korea \\ Byung Jun MiN \\ Department of Radiation Oncology, Chungbuk National University Hospital, Cheongju 28644, Korea \\ Kyungdon CHOI \\ RAON Users Association, Daejeon 34126, Korea \\ Ki-Whwan KIM \\ Department of Radiation Oncology, Chungnam National University Hospital, Daejeon 27478, Korea
}

DOI: $10.3938 / \mathrm{jkps.} 77.537$

The last author's name should be changed from "Ki-Whwan KIM" to "Ki-Hwan KIM", and the postal code should be changed from " 27478 " to " 35015 ".

*E-mail: wynpark@chungbuk.ac.kr 